\title{
Thymoma Versus Thymic Carcinoma: Differences in Biology Impacting Treatment
}

\author{
Ronan J. Kelly, MD, MBA
}

\begin{abstract}
A better understanding of the biology of both thymomas and thymic carcinomas has occurred in recent years thanks to advanced technologies such as comparative genomic hybridization, expression array analysis, and next-generation sequencing. Gene expression profiling and genomic clustering studies have shown that thymic tumors as classified by the 2004 WHO system do have different molecular features. Because of the rarity of these tumors, there is a paucity of high-quality clinical research data, and treatment decisions are often guided by the small amount of prospective trial data, retrospective series, and individual case reports. The literature does report on several advanced thymic tumors that have responded to new targeted agents, indicating that across the spectrum of thymic malignancies there may be clinically relevant molecular subsets. Genomic profiling distinguishes type B3 thymoma and thymic carcinoma from type $A$ and $B 2$ thymomas. Furthermore, type B2 thymomas can be separated from other subgroups in that it has a more distinctly lymphocytic component than the other groups in which epithelial cells predominate. The presence of KIT mutations in thymic carcinomas rather than in thymomas further adds to a growing body of evidence showing that underlying tumor biology may in the future lead to molecular classifications, which may enhance therapies for these rare tumors. (JNCCN 2013;11:577-583)
\end{abstract}

Thymic epithelial tumors account for approximately $20 \%$ of all mediastinal tumors. Nevertheless, they are rare compared with other malignancies, constituting only $0.2 \%$ to $1.5 \%$ of all solid tumors. ${ }^{1}$ The WHO classification system distinguishes thymomas (types $\mathrm{A}, \mathrm{AB}$, B1, B2, and B3) from thymic carcinomas (type C) based on the morphology of epithelial tumor cells (with increasing degree of atypia along the spectrum from type A to C), proportion of lymphocytic involvement, and resemblance to normal thymic tissue. Clinically, these diseases can also present differently with a large variety of autoimmune disorders, including myasthenia gravis (30\%) occurring in patients with thymoma, whereas patients with thymic carcinoma rarely if ever have autoantibody-induced phenomena. ${ }^{2}$ Surgery continues to be the most important therapeutic modality for early-stage disease, and a multidisciplinary approach incorporating surgery, radiation, and chemotherapy is recommended in advanced or recurrent disease. Research, however, has been hampered by the rarity of these tumors, which has led to a lack of international consensus surrounding appropriate histopathologic and staging criteria. Much debate has occurred regarding the limitations of the current histologic classifications with regard to both subtype definitions and consistency of diagnosis. The lack of established cell lines and animal models has hindered laboratory investigations, resulting in limited improvements in understanding of tumor biology. In the past decade, newer techniques such as comparative genomic hybridization (CGH), expression array analysis, and next-generation sequencing have resulted in incremental improvements in the understanding of these highly heterogenous tumors. This article focuses on the biological differences between thymomas and thymic carcinomas that may impact treatment decisions, and discusses targeted therapy trials performed to date in the advanced disease setting. 
Kelly

\section{Staging and Pathologic Classification of Thymomas and Thymic Carcinomas}

The most widely used system for staging thymic malignancies is the Masaoka staging system, which was originally developed in 1981 and focuses on the integrity of the thymic capsule, the presence of microor macroscopic invasion into adjacent structures, and metastatic spread. ${ }^{3}$ The WHO histologic classification of thymomas and thymic carcinomas, first published in 1999 and updated in 2004, ${ }^{4}$ has helped alleviate some of the prior confusion caused by the presence of several previous classification systems. Types A, AB, and B1 have an excellent overall survival rate of greater than $90 \%$ to $95 \%$ at 10 years. ${ }^{5}$ Five-year survival for types B2 and B3 and thymic carcinoma are $75 \%, 70 \%$, and $48 \%$, respectively. ${ }^{6}$ Thymic carcinomas account for fewer than $1 \%$ of thymic malignancies and are much more aggressive tumors than thymomas. ${ }^{7}$ Clinically thymic carcinomas are more likely to metastasize to the liver, lymph nodes, and bones compared with thymomas, which rarely spread beyond the site of origin. Several subtypes of thymic carcinoma exist, including squamous cell, basaloid, sarcomatoid, mucoepidermoid, papillary, lymphoepithelioma-like, and undifferentiated carcinomas. Knowledge of the tumor biology underlying the different phenotypes between thymomas and thymic carcinomas has improved in recent years.

\section{Immunologic Differences Between Thymomas and Thymic Carcinomas}

In addition to differing clinically, thymomas and thymic carcinomas have distinctive immunologic, genetic, and molecular characteristics. Morphologic differences are outlined in the WHO classification, but immunohistochemical markers such as expression of major histocompatability complex (MHC) class II or AIRE (autoimmune regulator gene) are becoming apparent. Approximately 30\% to $40 \%$ of patients with thymoma develop an autoimmune condition, $50 \%$ of which will be myasthenia gravis. ${ }^{8}$ Unlike those with thymomas, patients with thymic carcinomas rarely develop autoantibody-induced phenomena. The molecular basis underlying defective AIRE expression in thymoma versus thymic carcinoma is not understood. The AIRE gene is located on chromosome 21q22.3 and is considered important for ectopic expression of peripheral self- antigen and for central thymic T-cell education and deletion of autoreactive clones. ${ }^{9,10}$ Patients with thymomas have higher rates of autoantibodies to cytokines than patients with thymic carcinomas, ${ }^{11}$ and also have a higher risk of developing second malignancies, most notably, non-Hodgkin's lymphoma. A greater understanding of immunity and autoimmunity in thymoma is needed and may lead to future incorporation of immunotherapeutic strategies into treatment paradigms for thymomas.

\section{Molecular Biology Differences Between Thymomas and Thymic Carcinomas}

Cytogenetic studies have revealed chromosomal abnormalities in all histologic subtypes, including $\mathrm{t}(15 ; 19)$ translocations and 6p22-p25 deletions. ${ }^{6}$ The most frequent genetic alterations identified across the spectrum of thymomas occur on chromosome 6p21.3 (MHC locus) and 6q25.2-25.3.12-14 Although thymic carcinomas are a distinct entity in the WHO classification system, they do share some similarities with type B3 thymomas, most notably in the gain of 1q and loss of chromosome $6 .{ }^{13}$ Additional data from $\mathrm{CGH}$ analysis performed on thymic carcinomas have demonstrated frequent copy number gains of $17 q$ and 18 and loss of $3 p, 16 q$, and $17 p .^{13,15}$ The degree of genomic and recurrent copy number alterations increases from type A to type B3 and thymic carcinoma (Figure 1). Additional aberrations that have been described include multiple losses of genetic material and microsatellite instability in different chromosomes (3p22-24.3; 3p14.2 [FHIT gene locus]; 5q21 [APC gene]; 6p21; 6p21-22.1; 7p21-22; 8q11.21-23; 13q14 [RB gene]; and 17p13.1 [p53 gene]). ${ }^{15,16}$ In addition to the losses in the long arm of chromosome 6 , the loss of heterozygosity ( $\mathrm{LOH}$ ) on chromosome 5 (5q21; APC gene) may be the most significant. Inoue et $\mathrm{a}^{14}$ showed that alterations in chromosome 6 vary according to the WHO subtype (type A, 10\%; type AB, 12\%; type B, 20\%-26\%; thymic carcinoma, 35\%) and with clinical stage at diagnosis (stage I, 7\%; stage II, 27\%; stage III, 21\%; stage IV, 24\%). In this study, the authors found genetic aberrations on chromosome 6 in 31 of 40 thymoma cases evaluated $(77.5 \%)$, and these occurred in 5 separate hot spots. The most frequent LOHs $(48.6 \%)$ occurred in region $6 q 25.2$. Another hot spot showing $\mathrm{LOH}$ in $32.4 \%$ of tumors was located 

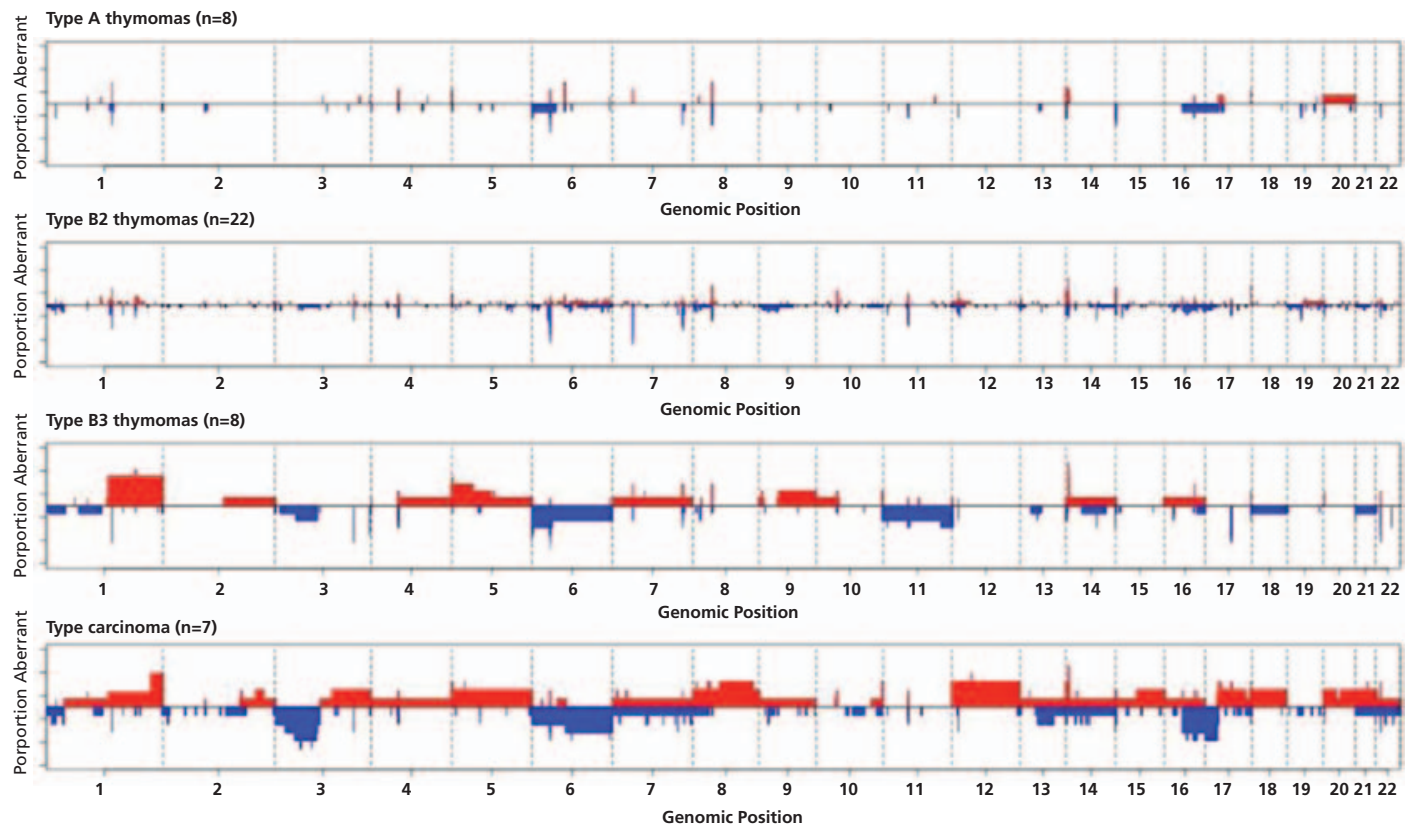

Figure 1 Correlation of thymic epithelia histotypes with specific genomic abnormalities. Red represents the frequency of copy number $(\mathrm{CN})$ gain, and blue represents the frequency of $\mathrm{CN}$ loss for 8 type A thymomas, 22 type B2 thymomas, 8 type B3 thymomas, and 7 thymic carcinomas. From Girard N, Shen R, Guo T, et al. Comprehensive genomic analysis reveals clinically relevant molecular distinctions between thymic carcinomas and thymomas. Clin Cancer Res 2009;15:6798; with permission.

on 6q25.2-25.3. The third hot spot (30\%) showing $\mathrm{LOH}$ appeared in region $6 \mathrm{p} 21.31$, including the MHC locus, and the fourth $(26.3 \%)$ was detected on 6q14.1-14.3. Some tumors (21.6\%) showed LOHs within a fifth hot spot on $6 \mathrm{q} 21$. Inoue et $\mathrm{a}^{14}$ concluded that several tumor suppressor genes on chromosome 6 seem to be involved in the pathogenesis of thymoma. MSI has also been described on chromosome 6 in 10\% of thymomas, more commonly in type B thymoma.

Girard et $\mathrm{al}^{15}$ performed array-based comparative genomic hybridization to identify potential recurrent copy number changes at the DNA level on 45 thymic tumor specimens. In this analysis, a hierarchical cluster algorithm revealed 2 major groups. Cluster 1 $(n=19)$ was associated with thymic carcinoma and type B3 thymoma and was characterized by multiple chromosomal aberrations. Cluster $2(n=26)$ was associated with type $\mathrm{A}$ and B2 thymomas and showed infrequent copy number alterations $\left(P<.001 ; \chi^{2}\right.$ test $)$. Interestingly, no association was seen between clinical stage and the presence of these genomic clusters. Further subdivision of cluster 1 was possible into cluster 1a $(n=9)$ with only type B3 thymomas and thymic carcinomas, characterized by chromosome 1 gain, and cluster $1 b(n=7)$ containing thymomas and thymic carcinomas sharing chromosome 6 loss. The group separately analyzed thymoma types A and $\mathrm{B}$ and thymic carcinoma to identify recurrent gene copy number alterations, but no significant results were identified.

In an effort to further understand the molecular biology of thymic malignancies and to progress beyond clinical and morphologic features, Badve et a ${ }^{17}$ performed whole-genome gene expression analysis and correlated their findings with outcomes in 34 patients with thymoma. In this study, 10 patients had stage I disease, 12 had stage II, 6 had stage III, and 6 had stage IV, and all patients had surgery with curative intent. The tumors were categorized into group 1 (types $A$ and $B ; n=9$ ), group 2 (types $B 1$ and B2; $n=19$ ), and group 3 (type B3; $n=6$ ). Unsupervised clustering of gene expression data identified 4 clusters of thymic tumors that showed significant correlation with histologic classification $(P=.002)$. Neither histology nor clusters correlated with clinical outcome. As a result of significant differential expression $(P<.01)$ with histologic groups in ANOVA analysis, the authors selected the top 15 upregulated and 15 downregulated genes to generate a supervised clustering heat map (Figure 2), indicating that gene expression analysis may separate these heteroge- 
Kelly

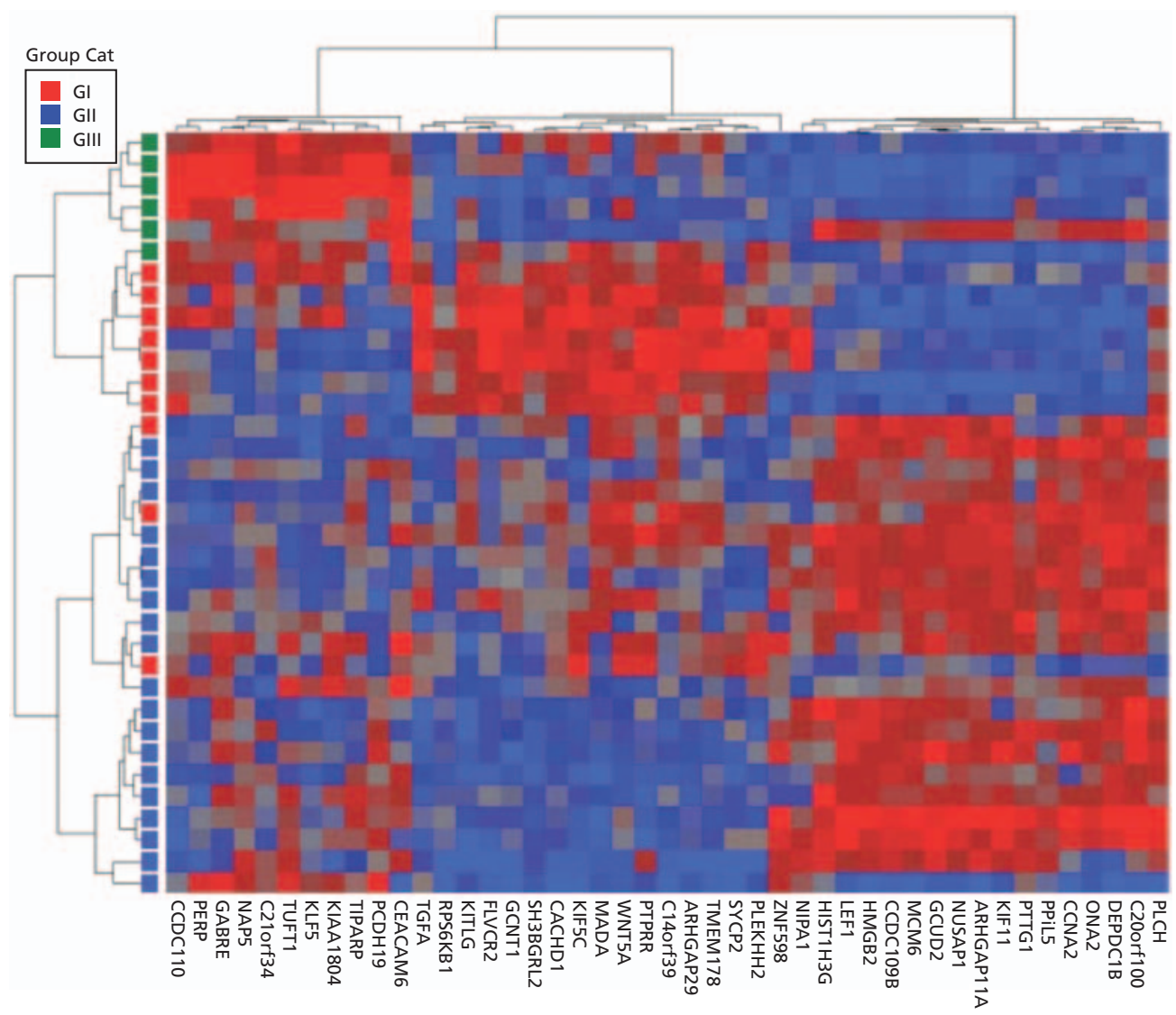

Figure 2 Supervised hierarchical clustering of fresh frozen thymomas based on histologic groups. This figure comprises 34 tumors. GI, group I (type AB thymomas); GII, group II (types B1-B2 thymomas); GIII, group III (type B3). From Badve S, Goswami C, Gokmen-Polar Y, et al. Molecular analysis of thymoma. PLoS One 2012;7:e42669; with permission.

neous tumors into separate clusters, which may have implications for future treatment strategies. In addition, the authors identified several genes associated with clinical behavior of thymic tumors, including stage, relapse, and metastasis, and some were confirmed using quantitative reverse transcription polymerase chain reaction (qRT-PCR). Among the top genes chosen, Aldo-keto reductase family 1 B10 (AKR1B10) and junctophilin-1 (JPH1) were upregulated in both stage III and IV disease. A hedgehog target gene, COL11A1, was downregulated in advanced-stage disease. AKR1B10 and JPH1 showed a 5.96-fold $(P=.05)$ and 3.01-fold $(P=.18)$ increase in metastasis-positive tumors compared with nonmetastatic disease, and COL11A1 was decreased 12.5-fold $(P=.11)$ in stage IV tumors. As a result of the differential expression levels of these genes, they were selected for validation by qRT-PCR, which confirmed the differential expression in the different phenotypes. These findings require external vali- dation in independent cohorts of cases, but may in the future suggest potential therapeutic targets. The presence of identifiable genetic aberrations in thymic malignancies has stimulated interest in targeted therapy trials in an effort to improve treatments of advanced disease.

\section{Targeted Therapy}

Personalized medicine is applicable to rare tumors such as thymomas and thymic carcinomas. Several small studies and case reports have evaluated targeted therapies, and ongoing trials are evaluating genetic alterations that may be predictive or prognostic in thymic malignancies (Table 1 ).

\section{KIT Pathway}

KIT is differentially expressed in thymic malignancies, with immunohistochemistry positivity occurring in up to $73 \%$ to $86 \%$ of thymic carcinomas, but limited overexpression is seen in thymomas, with 
Thymoma Versus Thymic Carcinoma

\begin{tabular}{|llll|}
\hline \multicolumn{2}{|c|}{ Table 1 Molecular Abnormalities in Thymoma and Thymic Carcinoma } \\
\hline Molecular Change/Oncogene & $\begin{array}{l}\text { Thymoma } \\
(\%)\end{array}$ & $\begin{array}{l}\text { Thymic Carcinoma } \\
(\%)\end{array}$ & Reference \\
\hline $\begin{array}{l}\text { C-KIT } \\
\quad \text { Overexpression (IHC) }\end{array}$ & $<5$ & $73-86$ & 18,19 \\
$\quad \begin{array}{l}\text { HER2 } \\
\quad \text { Overexpression (IHC) }\end{array}$ & 6 & 53 & 32 \\
$\begin{array}{l}\text { EGFR } \\
\quad \text { Gene amplification (FISH) } \\
\quad \text { Overexpression (IHC) }\end{array}$ & 20 & 25 & 27 \\
$\quad \begin{array}{l}\text { IGF-1R } \\
\text { Overexpression }\end{array}$ & 4 & $20-30$ & 19,26 \\
\hline
\end{tabular}

Abbreviations: EGFR, epidermal growth factor receptor; FISH, fluorescence in situ hybridization; IGF-1R, insulin-like growth factor-1 receptor; IHC, immunohistochemistry.

only $2 \%$ demonstrating positivity. ${ }^{18,19}$ It was originally believed that this difference in tumor biology would lead to a clear difference in treatment strategies for thymic carcinomas versus thymomas because of the fact that KIT is a recognized target in other tumor types, most notably gastrointestinal stromal tumors. Unfortunately, despite the high frequency of KIT expression in thymic carcinomas, the rate of KIT mutations remains low at $7 \%$ to $9 \%$. Four mutations have been described to date: the V560 deletion ${ }^{20}$ and L576P substitution, ${ }^{21}$ both found in exon 11 ; D820E mutation in exon $17^{22}$; and the H697Y mutation found in exon $14 .{ }^{15}$ A phase II trial evaluated imatinib in 7 patients with either type B3 thymoma $(n=2)$ or thymic carcinoma $(n=5)$. KIT expression was found in 1 of 4 samples on immunohistochemistry, and no mutations in c-KIT or platelet-derived growth factor receptor A (PDGFR-A) genes were demonstrated in 3 tumors examined. No responses were seen with imatinib therapy. ${ }^{23}$ Sunitinib may have activity in patients with thymic carcinoma harboring KIT mutations. Three of 4 patients experienced partial responses ranging from 2 to 18 or more months, and the other had prolonged stable disease lasting 22 months. ${ }^{24}$ Similarly, Girard et $\mathrm{al}^{15}$ showed that the $\mathrm{H} 697$ mutation is potently inhibited by both imatinib and sunitinib. Another case report documented disease activity in a patient with thymic carcinoma with a KIT D820E mutation (resistant to imatinib) treated with sorafenib, reporting a partial response in all metastatic sites and a progression-free survival of 15 months. ${ }^{22}$ Future decisions regarding the sensitivity of thymic carcinomas to KIT inhibi- tors will need to match the identified mutation to the correct targeted agent. Published sensitivities to date show that the V560 deletion and L576P substitution are sensitive to imatinib, the D820E mutation is sensitive to nilotinib, and the H697Y mutation is sensitive to sorafenib. ${ }^{16}$

\section{Epidermal Growth Factor Pathway}

Unlike lung cancer, somatic activating epidermal growth factor receptor (EGFR) mutations are extremely rare in thymic malignancies, except for a few isolated case reports in Asian patients. ${ }^{21,25}$ EGFR protein overexpression on immunohistochemistry is present in approximately $70 \%$ of thymomas and $30 \%$ of thymic carcinomas. ${ }^{19,26}$ No correlation is seen between EGFR staining and thymoma histologic type. EGFR gene amplification with fluorescence in situ hybridization (FISH) occurs in approximately 20\% of thymic malignancies, most notably in type B3 thymomas and thymic carcinomas, and is associated with more advanced stage and capsule invasion. ${ }^{27} \mathrm{~A}$ small study evaluated the efficacy of gefitinib, $250 \mathrm{mg}$ daily, in 26 previously treated patients with metastatic thymoma $(n=19)$ or thymic carcinoma $(\mathrm{n}=7),{ }^{28}$ showing no complete responses, 1 partial response lasting 5 months, and 14 stable diseases. Median time to progression was 4 months. None of the tumors analyzed with DNA sequencing had evidence of EGFR or KRAS mutations. A second phase II trial evaluated the efficacy and safety of the combination of erlotinib and bevacizumab in 18 (thymoma, 11; thymic carcinoma, 7) pretreated patients with progressive malignant thymic tumors. Standard-dose erlotinib, 150 $\mathrm{mg}$ daily, and bevacizumab, $15 \mathrm{mg} / \mathrm{kg}$ intravenously 
Kelly

per cycle, were repeated every 21 days. ${ }^{29}$ Sixty percent (11 patients) had stable disease, without any partial or complete responses, indicating that the combination of erlotinib and bevacizumab has limited activity in thymic malignancies. Cetuximab has been reported to have some activity, although these data are from 2 single case reports in which 1 patient overexpressed EGFR on immunohistochemistry and the other did not..$^{30,31} \mathrm{~A}$ phase II trial of neoadjuvant chemotherapy (cisplatin, doxorubicin, and cyclophosphamide) with cetuximab in patients with Masaoka stage II-IVA thymoma is currently recruiting patients (ClinicalTrials.gov identifier: NCT01025089). This trial will enroll 28 patients with locally advanced or recurrent disease and is expected to complete accrual at the end of 2013. Currently, evidence from the literature suggests that both EGFR tyrosine kinase inhibitors and monoclonal antibodies cannot be recommended for treating patients with thymic malignancies. Similarly, no cases of HER2 gene amplification by FISH have been detected, ${ }^{32}$ and no data are available to recommend HER2-targeted therapies in this disease setting.

\section{Vascular Endothelial Growth Factor Pathway}

Angiogenesis is thought to play an important role in thymomagenesis. Vascular endothelial growth factor (VEGF)-A and VEGF receptor (VEGFR)- 1 and -2 are overexpressed in both thymomas and thymic carcinomas, ${ }^{33,34}$ but data are limited regarding the efficacy of angiogenesis inhibitors in thymic malignancies. Low response rates have been seen with bevacizumab. ${ }^{29}$ Case reports involving sorafenib and sunitinib have highlighted the activity of these multikinase inhibitors, predominantly in thymic carcinomas. Activity was reported in a patient receiving sorafenib who had a missense mutation in exon 17 (D820E) of the c-KIT gene, ${ }^{22}$ as discussed earlier, and a second patient had prolonged stable disease ( $>9$ months) in a nonmutated but high immunohistochemistry-expressing tumor for KIT, p53, and VEGF.35 Angiogenesis inhibitors alone do not seem to have much activity in either thymomas or thymic carcinomas, but the multikinase inhibitors may have some activity in thymic carcinoma, although alternate pathway inhibition rather than a direct VEGF effect is the most likely explanation.

\section{Histone Deacetylase Inhibitors}

Histone deacetylase (HDAC) inhibitors have been evaluated in thymic tumors, most notably the panHDAC inhibitor belinostat, which demonstrated modest antitumor activity. In a phase I study of belinostat, a patient with thymoma experienced a minor response that lasted for 17 months on treatment. ${ }^{36} \mathrm{As}$ a result, the authors performed a phase II trial, whereby belinostat was given through intravenous infusion at $1 \mathrm{~g} / \mathrm{m}^{2}$ on days 1 through 5 of a 21-day cycle until disease progression. ${ }^{37}$ Forty-one patients were enrolled: 25 with thymoma and 16 with thymic carcinoma. Two partial responses in patients with in thymomas (response rate, 8\%; 95\% CI, 2.2\%-25\%), 25 stable diseases, and 13 progressions were seen. No responses were seen in patients with thymic carcinomas.

\section{Insulin-Like Growth Factor Pathway}

The insulin-like growth factor-1 (IGF-1)/IGF-1 receptor (IGF-1R) has been identified as a poor prognostic indicator in thymic malignancies. ${ }^{38,39}$ Expression of IGF-1R does differ between thymomas (4\%) and thymic carcinomas (37\%), indicating a possible difference in tumor biology that may be targetable. ${ }^{39}$ In a retrospective analysis, IGF-1R expression was less in types $A, A B$, and $B 1$ thymomas compared with types B2 and B3 and thymic carcinomas $(P<.001) .{ }^{39}$ The authors performed a phase II study of cixutumumab, an IGF-1R monoclonal antibody in patients with previously treated advanced thymic tumors. No activity was demonstrable in thymic carcinomas; however, the results for activity in thymomas are awaited. ${ }^{16}$

\section{Conclusions}

Tumors of the thymus are defined histologically by the 2004 WHO classification system; however, expression and genomic clustering results indicate that different molecular features exist across the spectrum of thymic tumors. Additionally, thymic carcinomas and type B3 thymomas do seem to be different from type A and B2 thymomas, indicating that a molecular classification may be more useful in future classification systems. The rarity of thymic malignancies has for years been seen as a hindrance in efforts to understand the biology of these tumors. Although large-scale phase III trials are not possible in advanced thymic malignancies, in the past decade researchers have begun to decipher the various molecular abnormalities that in the future may lead to more successful therapeutics. Although surgery continues to be the mainstay of treatment in operable cases, efforts are underway to develop genetic signa- 
Thymoma Versus Thymic Carcinoma

tures in the clinically relevant subsets of thymomas and thymic carcinomas to determine which patients will need which treatment. The International Thymic Malignancies Interest Group is fostering greater international collaborations in an effort to improve the outcomes for patients in the years ahead.

\section{References}

1. Engels EA. Epidemiology of thymoma and associated malignancies. J Thorac Oncol 2010;5(10 Suppl 4):S260-265.

2. Margaritora S, Cesario A, Cusumano G, et al. Thirty-five-year followup analysis of clinical and pathologic outcomes of thymoma surgery. Ann Thorac Surg 2010;89:245-252; discussion 252.

3. Masaoka A, Monden Y, Nakahara K, Tanioka T. Follow-up study of thymomas with special reference to their clinical stages. Cancer 1981;48:2485-2492.

4. Muller-Hermelink HK, Engel P, Kuo TT, et al. Tumours of the thymus. World Health Classification of Tumours, In: Travis WD, Brambilla E, Muller-Hermelink HK, Harris CC. Pathology \& Genetics: Tumours of the Lung, Pleura, Thymus and Heart. Lyon, France: IARC Press. 2004:146-248.

5. Chen G, Marx A, Chen WH, et al. New WHO histologic classification predicts prognosis of thymic epithelial tumors: a clinicopathologic study of 200 thymoma cases from China. Cancer 2002;95:420-429.

6. Herens C, Radermecker M, Servais A, et al. Deletion (6)(p22p25) is a recurrent anomaly of thymoma: report of a second case and review of the literature. Cancer Genet Cytogenet 2003;146:66-69.

7. Kuhn E, Wistuba II. Molecular pathology of thymic epithelial neoplasms. Hematol Oncol Clin North Am 2008;22:443-455.

8. Okumura $\mathrm{M}$, Fujii $\mathrm{T}$, Shiono $\mathrm{H}$, et al. Immunological function of thymoma and pathogenesis of paraneoplastic myasthenia gravis. Gen Thorac Cardiovasc Surg 2008;56:143-150.

9. Scarpino S, Di Napoli A, Stoppacciaro A, et al. Expression of autoimmune regulator gene (AIRE) and T regulatory cells in human thymomas. Clin Exp Immunol 2007;149:504-512.

10. Anderson MS, Venanzi ES, Chen Z, et al. The cellular mechanism of Aire control of T cell tolerance. Immunity 2005;23:227-239.

11. Burbelo PD, Browne SK, Sampaio EP, et al. Anti-cytokine autoantibodies are associated with opportunistic infection in patients with thymic neoplasia. Blood 2010;116:4848-4858.

12. Inoue $\mathrm{M}$, Starostik $\mathrm{P}$, Zettl $\mathrm{A}$, et al. Correlating genetic aberrations with World Health Organization-defined histology and stage across the spectrum of thymomas. Cancer Res 2003;63:3708-3715.

13. Zettl A, Strobel P, Wagner K, et al. Recurrent genetic aberrations in thymoma and thymic carcinoma. Am J Pathol 2000;157:257-266.

14. Inoue $\mathrm{M}$, Marx A, Zettl $\mathrm{A}$, et al. Chromosome 6 suffers frequent and multiple aberrations in thymoma. Am J Pathol 2002;161:1507-1513.

15. Girard N, Shen R, Guo T, et al. Comprehensive genomic analysis reveals clinically relevant molecular distinctions between thymic carcinomas and thymomas. Clin Cancer Res 2009;15:6790-6799.

16. Kelly RJ, Petrini I, Rajan A, et al. Thymic malignancies: from clinical management to targeted therapies. J Clin Oncol 2011;29:4820-4827.

17. Badve S, Goswami C, Gokmen-Polar Y, et al. Molecular analysis of thymoma. PLoS One 2012;7:e42669.

18. Pan CC, Chen PC, Chiang H. KIT (CD117) is frequently overexpressed in thymic carcinomas but is absent in thymomas. J Pathol 2004:202:375-381.

19. Henley JD, Cummings OW, Loehrer PJ Sr. Tyrosine kinase receptor expression in thymomas. J Cancer Res Clin Oncol 2004;130:222-224.
20. Strobel P, Hartmann M, Jakob A, et al. Thymic carcinoma with overexpression of mutated KIT and the response to imatinib. N Engl J Med 2004;350:2625-2626.

21. Yoh $K$, Nishiwaki $Y$, Ishii $G$, et al. Mutational status of EGFR and KIT in thymoma and thymic carcinoma. Lung Cancer 2008;62:316-320.

22. Bisagni $G$, Rossi $G$, Cavazza $A$, et al. Long lasting response to the multikinase inhibitor bay 43-9006 (Sorafenib) in a heavily pretreated metastatic thymic carcinoma. J Thorac Oncol 2009;4:773-775.

23. Giaccone G, Rajan A, Ruijter R, et al. Imatinib mesylate in patients with WHO B3 thymomas and thymic carcinomas. J Thorac Oncol 2009;4:1270-1273.

24. Strobel P, Bargou R, Wolff A, et al. Sunitinib in metastatic thymic carcinomas: laboratory findings and initial clinical experience. $\mathrm{Br} \mathrm{J}$ Cancer 2010;103:196-200.

25. Yamaguchi $\mathrm{H}$, Soda $\mathrm{H}$, Kitazaki $\mathrm{T}$, et al. Thymic carcinoma with epidermal growth factor receptor gene mutations. Lung Cancer 2006;52:261-262.

26. Suzuki E, Sasaki H, Kawano O, et al. Expression and mutation statuses of epidermal growth factor receptor in thymic epithelial tumors. Jpn J Clin Oncol 2006;36:351-356.

27. Ionescu DN, Sasatomi E, Cieply K, et al. Protein expression and gene amplification of epidermal growth factor receptor in thymomas. Cancer 2005;103:630-636.

28. Kurup A, Burns M, Dropcho S, et al. Phase II study of gefitinib treatment in advanced thymic malignancies [abstract]. J Clin Oncol 2005;23(Suppl):Abstract 7068

29. Bedano PM, Perkins S, Burns M, et al. A phase II trial of erlotinib plus bevacizumab in patients with recurrent thymoma or thymic carcinoma [abstract]. J Clin Oncol 2008;26(Suppl):Abstract 19087.

30. Farina G, Garassino MC, Gambacorta M, et al. Response of thymoma to cetuximab. Lancet Oncol 2007;8:449-450.

31. Palmieri G, Marino M, Salvatore M, et al. Cetuximab is an active treatment of metastatic and chemorefractory thymoma. Front Biosci 2007;12:757-761.

32. Pan CC, Chen PC, Wang LS, et al. Expression of apoptosis-related markers and HER-2/neu in thymic epithelial tumours. Histopathology 2003;43:165-172.

33. Cimpean AM, Raica M, Encica S, et al. Immunohistochemical expression of vascular endothelial growth factor A (VEGF), and its receptors (VEGFR1, 2) in normal and pathologic conditions of the human thymus. Ann Anat 2008;190:238-245.

34. Sasaki H, Yukiue H, Kobayashi $Y$, et al. Elevated serum vascular endothelial growth factor and basic fibroblast growth factor levels in patients with thymic epithelial neoplasms. Surg Today 2001;31:10381040.

35. Li XF, Chen Q, Huang WX, Ye YB. Response to sorafenib in cisplatinresistant thymic carcinoma: a case report. Med Oncol 2009;26:157160.

36. Steele NL, Plumb JA, Vidal L, et al. A phase 1 pharmacokinetic and pharmacodynamic study of the histone deacetylase inhibitor belinostat in patients with advanced solid tumors. Clin Cancer Res 2008;14:804810 .

37. Giaccone G, Rajan A, Berman A, et al. Phase II study of belinostat in patients with recurrent or refractory advanced thymic epithelial tumors. J Clin Oncol 2011;29:2052-2059.

38. Girard N, Teruya-Foldstein J, Payabyab EC, et al. Insulin-like growth factor-1 receptor expression in thymic malignancies. J Thorac Oncol 2010;5:1439-1446.

39. Zucali PA, Petrini I, Lorenzi E, et al. Insulin-like growth factor-1 receptor and phosphorylated AKT-serine 473 expression in 132 resected thymomas and thymic carcinomas. Cancer 2010;116:4686-4695. 